\title{
Highly Elevated Quantitative D-Dimer Assay Values Increase the Likelihood of Venous Thromboembolism
}

\author{
Samuel Francis ${ }^{1}$ Alexander Limkakeng ${ }^{1}$ Hui Zheng ${ }^{2}$ Judd Hollander ${ }^{3}$ Gregory Fermann ${ }^{4}$ \\ Blair Alden Parry ${ }^{5}$ Frank Lovecchio ${ }^{6} \quad$ Nikos Werner $^{7} \quad$ Sebastian Schellong $^{8} \quad$ Christopher Kabrhel $^{5}$ \\ ${ }^{1}$ Division of Emergency Medicine, Duke University Hospital, Durham, \\ North Carolina, United States \\ ${ }^{2}$ Department of Biostatistics, Massachusetts General Hospital, \\ Address for correspondence Samuel Francis, MD, DUMC 3096 \\ 2301 Erwin Road, Durham, NC 27710, United States \\ Boston, Massachusetts, United States \\ (e-mail: samuel.francis@duke.edu). \\ ${ }^{3}$ Department of Emergency Medicine, Jefferson University, \\ Philadelphia, Pennsylvania, United States \\ ${ }^{4}$ Department of Emergency Medicine, University of Cincinnati, \\ Cincinnati, Ohio, United States \\ ${ }^{5}$ Department of Emergency Medicine, Center for Vascular \\ Emergencies, Massachusetts General Hospital, Boston, \\ Massachusetts, United States \\ ${ }^{6}$ Department of Emergency Medicine, Maricopa Hospital, Phoenix, \\ Arizona, United States \\ 7 International Center for Cardiovascular Interventions, Heart Center \\ Bonn, Medizinischen Klinik und Poliklinik II, Bonn, Germany \\ ${ }^{8}$ Städtisches Klinikum Dresden, Medizinische Klinik, Dresden, \\ Germany \\ TH Open 2019;3:e2-e9.
}

\begin{abstract}
Keywords

- venous thrombosis

- deep vein thrombosis

- pulmonary embolism

Objectives In patients with suspected venous thromboembolism (VTE), the D-dimer assay is commonly utilized as part of the workup. The assay is primarily used to determine whether to proceed with radiographic imaging. We compared D-dimer levels in patients suspected of having VTE. We hypothesized that higher D-dimer values predict a higher likelihood of subsequent VTE diagnosis.

Methods We conducted a secondary analysis of a multinational, prospective observational study of low- to intermediate-risk adult patients presenting to the emergency department with suspicion of VTE. Demographic and clinical data were collected in a structured manner. Advanced imaging including ultrasound, computed tomography (CT) pulmonary angiography, and ventilation/perfusion scanning was obtained at the discretion of the treating physicians. Imaging was evaluated by board-certified radiologists in real time. D-dimer values' bins were evaluated using a logistic regression model.

Results We evaluated 1,752 patients for suspected deep vein thrombosis (DVT), with 191 (10.4\%) DVT positive. We evaluated 1,834 patients for suspected pulmonary embolism (PE), with 108 (5.9\%) PE positive. Higher D-dimer values in both groups were associated with higher likelihood of subsequent VTE diagnosis, with D-dimer values $>3,999 \mathrm{ng} / \mathrm{mL}$ in both groups having the highest incidence of VTE. More than $50 \%$ of those patients were VTE positive.
\end{abstract}

received

April 30, 2018

accepted after revision

November 29, 2018
DOI https://doi.org/

$10.1055 / \mathrm{s}-0038-1677029$.

ISSN $2512-9465$. (c) 2019 Georg Thieme Verlag KG

Stuttgart · New York
License terms

(c) (1) 
Conclusions Increasing D-dimer values predict increased likelihood of being found VTE positive in this patient population. Among those in the highest D-dimer category, $>3,999 \mathrm{ng} / \mathrm{mL}$, over half of patients were VTE positive. Further research could determine additional nuance in D-dimer as a tool to work up suspected VTE.

\section{Introduction}

D-dimer testing in the workup of patients with potential venous thromboembolism (VTE) is commonplace. ${ }^{1-7}$ When used appropriately, D-dimer testing has demonstrated a reduction in radiographic imaging, decreased emergency department (ED) length of stay, and decreased total health care costs in appropriately risk-stratified groups. ${ }^{8}$ However, some studies have demonstrated an increase in workups and radiographic imaging for VTE with the introduction of the Ddimer assay without an associated increase in diagnosis. ${ }^{9}$

Since its implementation, the D-dimer has been utilized as a dichotomous test. When used in this manner, the Ddimer has a specificity at or below $50 \%{ }^{1,10}$ Studies in recent years have sought to adjust dichotomous D-dimer thresholds based on different criteria such as: age, clinician's pretest probability, or pregnancy. ${ }^{11-13}$ In each case, however, the Ddimer result is still used dichotomously; that is, below a certain threshold the test is considered negative and above the threshold it is considered positive. There have been several studies demonstrating that $\mathrm{D}$-dimer values can be used as continuous variables to predict likelihood of pulmonary embolism (PE). These studies have shown that a patient's chance of having PE increases with rising D-dimer concentration. ${ }^{14-16}$ However, there have not been any studies to date evaluating use of continuous D-dimer values in patients with suspected deep vein thrombosis (DVT). One study found a D-dimer value greater than $3.6 \mu \mathrm{g} / \mathrm{mL}$ increased the likelihood of subsequent DVT diagnosis. ${ }^{17}$ To date, there have not been any studies specifically examining the upper value D-dimer concentrations in the risk stratification of patients with suspected DVT.

In this secondary analysis of a prospective observational study of a D-dimer assay performed at multiple centers across the United States and Europe, there are two hypotheses. First, we hypothesize that the likelihood of DVT and PE increases linearly with rising D-dimer values. Second, using a bayesian approach, there are $\mathrm{D}$-dimer values above which a single negative radiographic interrogation (ultrasound, computed tomography pulmonary angiography [CTPA], or ventilation/perfusion [VQ]) no longer has sufficient posttest probably to avoid further evaluation in select cases using current recommended guidelines. ${ }^{18}$

\section{Methods}

\section{Setting}

We performed a secondary analysis of a prospective, observational study of D-dimer testing in consecutive ED patients with suspected VTE from 23 centers (17 USA, 6 Europe). ${ }^{19}$
The primary study was performed to determine the test characteristics of two available D-dimer assays (VIDAS Ddimer and Innovance D-dimer) using standard and ageadjusted cutoffs. All participating centers had the capability for CTPA, VQ scanning, venous ultrasound, and peripheral venography. Siemens Healthcare Diagnostics (Newark, Delaware, United States) was the sponsor of the study. Siemens had no role in analyzing or interpreting the data. The institutional review board (IRB) of each participating institution approved the study prior to participation and enrollment of any subjects.

\section{Selection of Participants}

Eligible individuals were 18 years and older who presented to an ED or outpatient clinic with suspected VTE and received objective testing by the treating clinician. D-dimer samples using the Innovance D-dimer assay were drawn after the decision was made by the clinician to test for DVT or PE but prior to results of the diagnostic workup. These individuals needed to be capable of providing informed consent. As part of their usual medical care, all participants received objective testing for VTE. Patients with possibility of recurrent VTE were included in the study. We excluded patients if they had high pretest probability for VTE (Wells' PE score $>6$ or Wells' DVT score $\geq 2$ ). Our other exclusion criteria included pregnancy and anticoagulation use for $>24$ hours prior to blood sample collection. We obtained written consent for all patients by trained research staff.

\section{Data Collection}

After patient consent, we collected baseline demographic data, including gender, race, age, Wells' pretest probability score for DVT or PE, D-dimer value, result of imaging procedure(s), outcome at 3-month follow-up, and final diagnosis. The data were collected by trained research staff. Resident and attending physicians gathered the data and calculated the Wells DVT and Wells PE scores to generate a pretest probability. We collected blood samples and pretest probability was assessed before the patient's usual care diagnostic test results had returned. Blood samples were drawn into $3.2 \%$ sodium citrate tubes. Within 4 hours of collection, samples were centrifuged to platelet-free plasma, transferred to microtubes, and frozen to $\leq-70^{\circ} \mathrm{C}$. The D-dimer concentrations used for this study were measured by the Innovance D-dimer platform (Siemens Healthcare Diagnostics, Newark, Delaware, United States) on the CS-5100 system at a central laboratory. D-dimer tests ordered as part of usual clinical care were not used for this analysis to maintain consistency across centers. 


\section{Outcome Measures}

Participants underwent a structured evaluation for VTE consistent with local usual care protocols. We considered patients to have a PE if their CTPA demonstrated a filling defect in a pulmonary artery or if a VQ scan was read as high probability for PE. If a patient was low or intermediate pretest probability for VTE but did not have imaging performed but had a negative D-dimer performed by the hospital's clinical laboratory, we considered them to have ruled out for VTE. We considered patients to have a DVT if a thrombus in a deep venous leg vein proximal to, or at the level of, the calf was found by either venous ultrasound or contrast venography. Patients with possibility of recurrent VTE were included in the study. For those patients, they were considered to be PE or DVT positive if imaging demonstrated clot in a new location or extension of a previously documented DVT. All radiologic studies were interpreted by board-certified radiologists.

The D-dimer categories were decided after analyzing the total number of patients included in specific "bins." By maintaining "bins" of $1,000 \mathrm{ng} / \mathrm{mL}$ beyond a D-dimer concentration of $1,000 \mathrm{ng} / \mathrm{mL}$, we were able to maintain an appropriate number of patients to reduce the risk of random chance causing the outcome. We decided $>3,999 \mathrm{ng} / \mathrm{mL}$ was the upper level "bin" so as to maintain an appropriate number of patients in each group.

Three months after the index visit, we performed a phone call follow-up with patients and we also reviewed their medical records. Patients with an initial negative workup for VTE were considered to have a PE or DVT during their 3month follow-up if they reported a diagnosed DVT or PE in the interval. Patients were considered lost to follow-up if they did not respond to five follow-up phone calls. For analysis purposes, these patients were not considered to have hemodynamically significant DVT or PE as long as they had negative criterion standard in their index visit.

\section{Statistical Analysis}

Demographic data are reported as means with standard deviations (SD) or simple percentages. Standard methods were utilized for calculating characteristics of D-dimer values such as odds ratios. Patients were analyzed in Ddimer bins of the following values: a logistic regression model was constructed using D-dimer level as predictor variable and VTE occurrence as outcome, while controlling for several variables. In patients with DVT, we controlled for gender, race, and the individual components of the Wells DVT score. In patients with PE, we controlled for age as well as the individual components of Wells' PE score. Data were analyzed using SAS version 9.4 (SAS Institute, Cary, North Carolina, United States). Adjusted odds ratios (ORs) were utilized for the D-dimer bins because they were derived from multivariable components as noted above.

\section{Results}

In total, 3,586 patients were evaluated for VTE; of these, 1,752 were evaluated for DVT and 1,834 were evaluated for PE (-Fig. 1). Among patients evaluated for DVT, the DVT prevalence was $10.9 \%(191 / 1752)$. Seventy-eight (4.4\%) had a calf DVT, and 113 (6.4\%) had a proximal DVT. Of the 191 DVTpositive patients, $4(0.2 \%)$ patients were considered to be DVT negative on their index visit and were subsequently found to be DVT positive at follow-up. The mean age was $53.1 \pm 16.2$ years, and $710(40.5 \%)$ were male.

There were 743 patients in the DVT cohort with a Ddimer $<500 \mathrm{ng} / \mathrm{mL}$. Eighteen (2.4\%) of these were found to be DVT positive. The average Wells' DVT score in these patients was 0.78 (SD 1.06). Of the 18 patients that were DVT positive, 9 had an isolated calf DVT. All 18 were diagnosed on the index visit. Nine out of 18 (50\%), had a prior history of DVT by Wells' DVT score.

Among patients evaluated for PE, the PE incidence was $5.9 \%(108 / 1834)$. In total, 101 (5.5\%) had a segmental or larger PE on imaging. Seven $(0.4 \%)$ were found to have a subsegmental PE. Of the 108 PE-positive patients, 7 (0.4\%) patients were considered to be PE negative on their index visit and were subsequently found to be PE positive at followup. The mean age was $47.4 \pm 15.8$ years, and 676 (36.9\%) were male. Additional demographic information is given in - Table 1. For the purposes of this analysis, those lost to follow-up were presumed to be VTE negative.

There were 962 patients in the PE cohort with a D-dimer $<500 \mathrm{ng} / \mathrm{mL}$. Three $(0.3 \%)$ of these were found to be PE positive. In those PE patients, the average Wells' PE score was 2.33. This is considered an intermediate-risk Wells' PE score. All three were diagnosed on their index visit.

D-dimer values were obtained on all patients. - Table 2 shows that the OR for DVT rises with progressively higher Ddimer concentrations to a maximum of 52 (95\% confidence interval [CI], 27-99) in patients with a D-dimer > 3,999 ng/ $\mathrm{mL}$. Utilizing a logistic regression model, the adjusted OR for D-dimer value in DVT as a continuous variable was 1.34 (95\% CI, 1.26-1.42), $p<0.0001$. The proportion of DVT diagnoses by D-dimer category is shown in -Fig. 2.

- Table 3 shows that the OR for PE rises with progressively higher D-dimer concentrations, to a maximum of 221 (95\% $\mathrm{CI}, 65-752$ ) in patients with a D-dimer $>3,999 \mathrm{ng} / \mathrm{mL}$. At the highest D-dimer values, more than half of the patients were ultimately diagnosed with VTE. Using a logistic regression model, the adjusted OR for D-dimer value in PE as a continuous variable was 1.31 (95\% CI, 1.23-1.40), $p<0.0001$. The rates of PE versus D-dimer in the various numerical categories are shown in -Fig. 2.

-Figs. 3 and $\mathbf{4}$ demonstrate the receiver operating characteristic (ROC) curves generated for patients with suspected PE and DVT, respectively, when utilizing the D-dimer as an initial test. The area under the curve for both PE and DVT was high, 0.910 (0.882-0.937) and 0.823, respectively.

In the highest D-dimer category, > 3,999 ng/mL, by eliminating patients with active cancer and/or recent surgery/ bedridden by Wells' DVT criteria, we found 52/95 patients, $54.7 \%$ (95\% CI, 44.2-64.9\%), to be DVT positive. In the patients with suspected PE in the highest D-dimer category, eliminating those with treated malignancy and/or with immobility or recent surgery by Wells' PE criteria left $38 / 67$ patients, 56.7\% (95\% CI, 44.1-68.6\%), PE positive. 


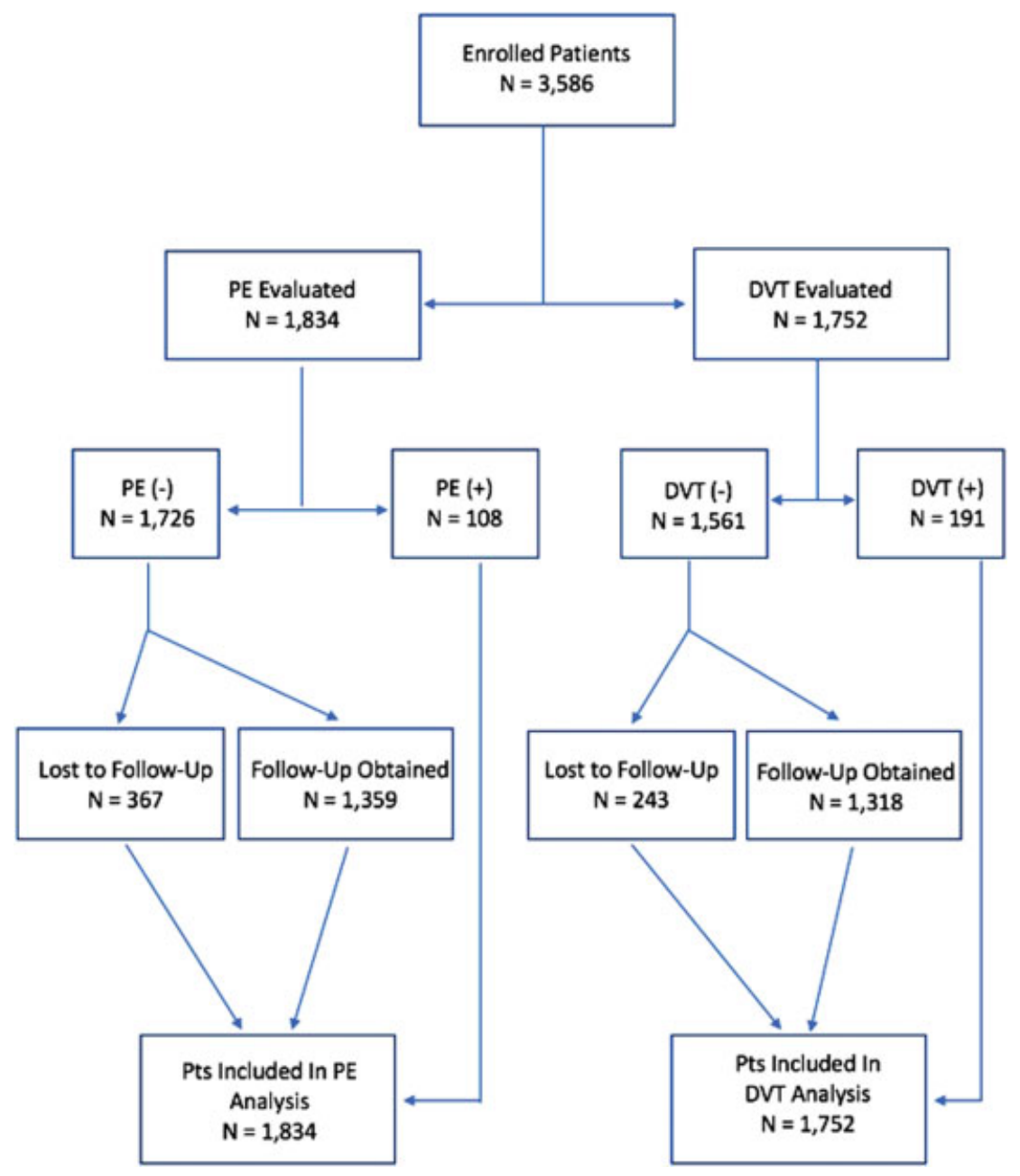

Fig. 1 Enrollment.

Table 1 Characteristics of patients evaluated for VTE

\begin{tabular}{|c|c|c|c|c|c|c|}
\hline & \multicolumn{3}{|c|}{ DVT $(N=1,752)$} & \multicolumn{3}{|c|}{$\mathrm{PE}(N=1,834)$} \\
\hline & $N$ & $\%$ & SD & $N$ & $\%$ & SD \\
\hline Age (mean) & - & 53.1 & 16.2 & - & 47.7 & 15.8 \\
\hline Gender (male) & 710 & 40.5 & - & 676 & 36.9 & - \\
\hline \multicolumn{7}{|l|}{ Race } \\
\hline White & 1,172 & 66.9 & - & 1,081 & 58.9 & - \\
\hline Black & 475 & 27.1 & - & 553 & 30.2 & - \\
\hline Hispanic & 79 & 4.5 & - & 145 & 7.9 & - \\
\hline Other & 14 & 0.8 & - & 37 & 2.0 & - \\
\hline Asian & 12 & 0.7 & - & 18 & 1.0 & - \\
\hline \multicolumn{7}{|c|}{ Wells' score category } \\
\hline Low & 576 & 32.9 & - & 1,175 & 64.1 & - \\
\hline Intermediate & 559 & 31.9 & - & 658 & 35.9 & - \\
\hline Unknown & 617 & 35.2 & - & 1 & 0.1 & - \\
\hline
\end{tabular}


Table 2 Proportion of DVT-positive and DVT-negative patients by D-dimer result

\begin{tabular}{|c|c|c|c|}
\hline $\begin{array}{l}\text { D-dimer } \\
\text { (ng/mL) }\end{array}$ & $\begin{array}{l}\text { Patients } \\
\text { (\#) }\end{array}$ & Percentage & aOR $(95 \% \mathrm{Cl})$ \\
\hline \multicolumn{4}{|l|}{$<500$} \\
\hline DVT $(+)$ & 18 & 2.4 & \multirow[t]{2}{*}{ Reference } \\
\hline DVT $(-)$ & 725 & 97.6 & \\
\hline \multicolumn{4}{|l|}{ 500-999 } \\
\hline DVT $(+)$ & 28 & 6.5 & \multirow[t]{2}{*}{$3.3(1.7-6.1)$} \\
\hline DVT (-) & 401 & 93.5 & \\
\hline \multicolumn{4}{|c|}{$1,000-1,999$} \\
\hline DVT $(+)$ & 34 & 13.0 & \multirow[t]{2}{*}{$6.9(3.7-12.8)$} \\
\hline DVT $(-)$ & 228 & 87.0 & \\
\hline \multicolumn{4}{|c|}{ 2,000-2,999 } \\
\hline DVT $(+)$ & 20 & 17.5 & \multirow[t]{2}{*}{$10.8(5.2-22.4)$} \\
\hline DVT $(-)$ & 94 & 82.5 & \\
\hline \multicolumn{4}{|c|}{$3,000-3,999$} \\
\hline DVT $(+)$ & 20 & 32.3 & \multirow[t]{2}{*}{$24.6(11.2-54.3)$} \\
\hline DVT $(-)$ & 42 & 67.7 & \\
\hline \multicolumn{4}{|l|}{$>3,999$} \\
\hline DVT $(+)$ & 71 & 50.0 & \multirow[t]{2}{*}{$51.7(26.9-99.2)$} \\
\hline DVT $(-)$ & 71 & 50.0 & \\
\hline
\end{tabular}

Table 3 Proportion of PE-positive and PE-negative patients by D-dimer result

\begin{tabular}{|c|c|c|c|}
\hline $\begin{array}{l}\text { D-dimer } \\
\text { (ng/mL) }\end{array}$ & $\begin{array}{l}\text { Patients } \\
\text { (\#) }\end{array}$ & Percentage & aOR $(95 \% \mathrm{Cl})$ \\
\hline \multicolumn{4}{|l|}{$<500$} \\
\hline $\mathrm{PE}(+)$ & 3 & 0.3 & \multirow[t]{2}{*}{ Reference } \\
\hline $\mathrm{PE}(-)$ & 959 & 99.7 & \\
\hline \multicolumn{4}{|l|}{ 500-999 } \\
\hline $\mathrm{PE}(+)$ & 11 & 2.8 & \multirow[t]{2}{*}{$7.2(2.0-26.1)$} \\
\hline $\mathrm{PE}(-)$ & 387 & 97.2 & \\
\hline \multicolumn{4}{|c|}{$1,000-1,999$} \\
\hline $\mathrm{PE}(+)$ & 18 & 7.7 & \multirow[t]{2}{*}{$17.7(5.1-61.7)$} \\
\hline $\mathrm{PE}(-)$ & 217 & 92.3 & \\
\hline \multicolumn{4}{|c|}{$2,000-2,999$} \\
\hline $\mathrm{PE}(+)$ & 13 & 14.4 & \multirow[t]{2}{*}{$37.4(10.2-138.1)$} \\
\hline $\mathrm{PE}(-)$ & 77 & 85.6 & \\
\hline \multicolumn{4}{|c|}{$3,000-3,999$} \\
\hline $\mathrm{PE}(+)$ & 8 & 19.0 & \multirow[t]{2}{*}{42.4 10.2-175.9) } \\
\hline $\mathrm{PE}(-)$ & 34 & 81.0 & \\
\hline \multicolumn{4}{|l|}{$>3,999$} \\
\hline $\mathrm{PE}(+)$ & 55 & 51.4 & \multirow[t]{2}{*}{$221.5(65.2-753.0$} \\
\hline $\mathrm{PE}(-)$ & 52 & 48.6 & \\
\hline
\end{tabular}

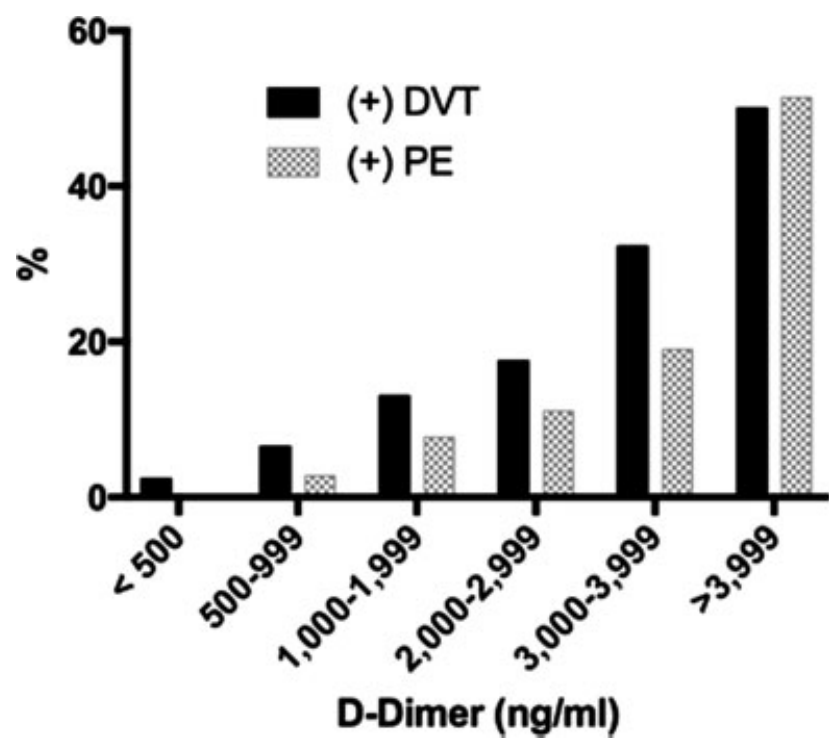

Fig. 2 DVT- and PE-positive patients based on D-dimer values.

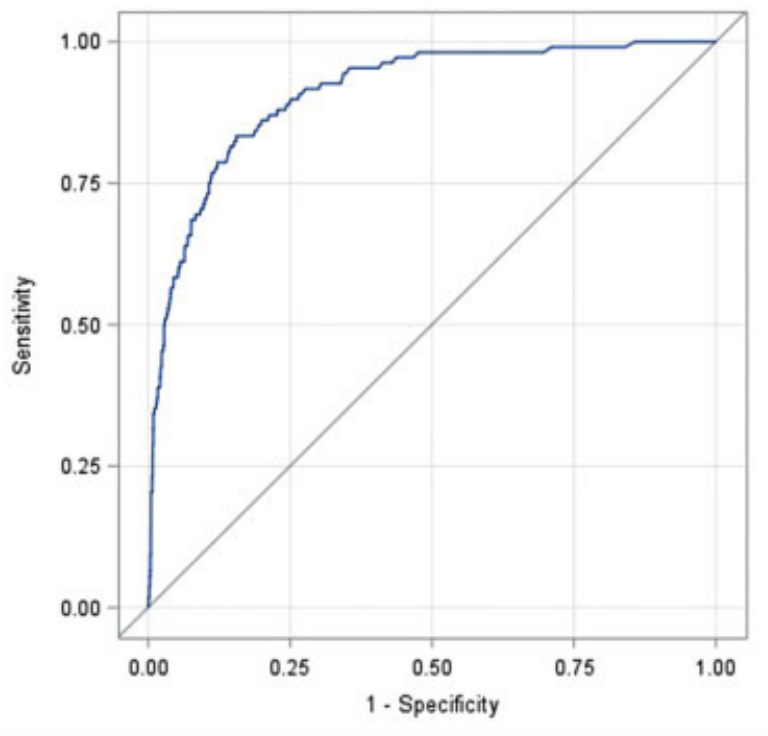

Fig. 3 ROC curve for D-dimer with regard to likelihood of PE. Area under curve is 0.910 .

\section{Discussion}

In this multicenter international observational study, we observed an increasing rate of VTE with increasing D-dimer levels. There appears to be value in utilizing the D-dimer in a more nuanced approach to VTE workup beyond its current dichotomous use. There does not appear to be an "absolute cutoff" beyond which meaningful information is no longer provided. Future decision instruments may be able to incorporate the $\mathrm{D}$-dimer value as a spectrum rather than a dichotomous cutoff in developing pretest probabilities for patients with suspected VTE.

Patients with a D-dimer value $<500 \mathrm{ng} / \mathrm{mL}$ had clinically apparent DVT and PE prevalence rates of 2.4 and $0.3 \%$, respectively. Given the risks of radiation, increased length 


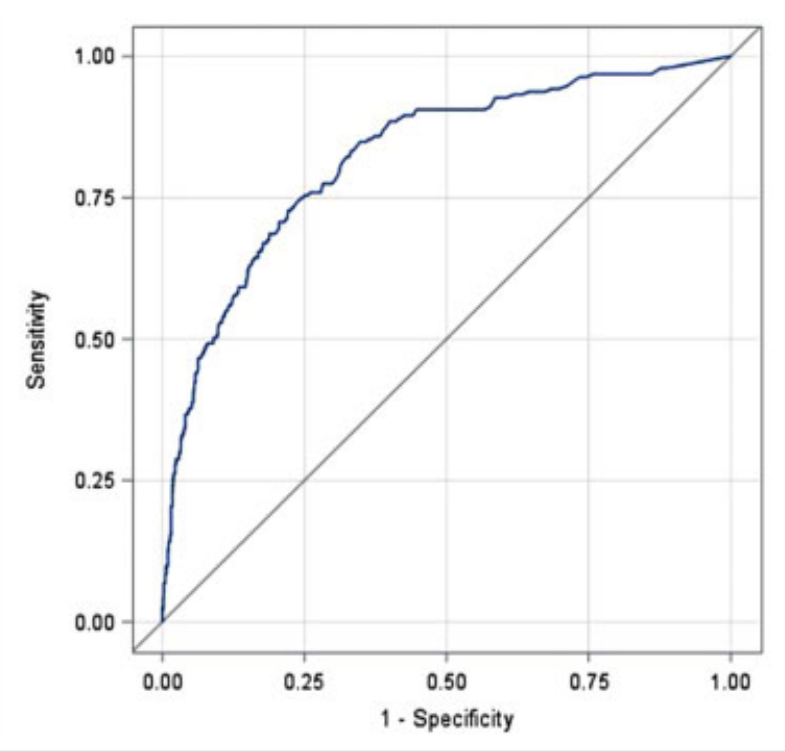

Fig. 4 ROC curve for D-dimer in patients with suspected DVT. The area under the curve was 0.823 .

of stays, and increased costs associated with additional workups, ${ }^{8}$ the data support no further VTE workup if the D-dimer returns with a normal value. It is important to recognize that patients with appropriately low pretest probability for PE should undergo D-dimer testing to reduce unnecessary radiation exposure. Studies have shown that D-dimer testing is bypassed in patients that meet criteria for initial D-dimer testing as part of the workup in favor of radiographic imaging. ${ }^{20}$ The data demonstrated here reiterate that individuals who are below the threshold D-dimer value of $<500 \mathrm{ng} / \mathrm{mL}$ should have their workup for VTE ceased if the test was ordered in the appropriate risk stratification group.

In this study, we found a clear relationship between increasing D-dimer values and likelihood of VTE diagnosis. This is the first study to evaluate this connection in patients with suspected DVT. In those patients at the highest D-dimer values ( $>3,999 \mathrm{ng} / \mathrm{mL}$ in our study), over $50 \%$ were found to be VTE positive. With the known false-negative rate for both DVT ultrasound and CTPA or VQ imaging for PE, clinicians should consider additional workup in those patients with Ddimer values in this range and either nondiagnostic or negative initial testing. ${ }^{21,22}$ Empiric anticoagulation has been recommended by some experts for patients initially deigned high risk for VTE prior to completion of imaging studies. ${ }^{23}$

In the $>3,999 \mathrm{ng} / \mathrm{mL}$ cohort of PE-positive patients, if we take the reported sensitivities and specificities of CTPA and VQ as noted in PIOPED II, we are able to calculate a negative likelihood ratio for both studies. ${ }^{24}$ CTPA has a sensitivity of $83 \%$ and a specificity of $96 \%$. VQ has a sensitivity of $77.4 \%$ and a specificity of $97.7 \%$. The negative likelihood ratios of CTPA and VQ are therefore 0.177 and 0.188 , respectively. With the prevalence of disease in the highest $\mathrm{D}$-dimer cohort being $50 \%$, therefore, a negative imaging study of either CTPA or VQ scanning leads to posttest probabilities of $15 \%$ for CTPA and $18.8 \%$ for VQ scan. For the clinician at the bedside, this means that one has to be attentive to the individual patient and recognize the risk of false-negative imaging findings in this group. The high sensitivity and specificity of venous ultrasound for proximal DVT, 96.5 and $94.3 \%$, respectively, ${ }^{25}$ reveal that negative imaging studies have a much lower posttest probability in the highest D-dimer cohort in those suspected of DVT. A presumed prevalence of $50 \%$ in the $>3,999 \mathrm{ng} / \mathrm{mL}$ category of D-dimer coupled with a negative likelihood ratio of 0.036 for venous ultrasound leads to a posttest probability of $3.6 \%$ with a negative DVT ultrasound.

Our dataset presents the spectrum of patients with suspected VTE at low and intermediate pretest probability. While much scrutiny has focused of the lower D-dimer levels, the cohort of patients with high D-dimer presents different challenges in risk stratification. When patients initially thought to be at low or moderate pretest probability for PE subsequently have high D-dimer values $>3,999 \mathrm{ng} / \mathrm{mL}$, the diagnostic strategy still includes CTPA, VQ or venous duplex ultrasonography. However, if these studies are negative or nondiagnostic, consideration should be given to further testing. One possibility is that the ultrasound or CTPA is falsely negative. As such, additional testing with angiography or perfusion scintigraphy may be options. Alternatively, the location of the thrombosis may be in an atypical location such as the pelvic, central nervous system, upper extremities, or jugular vessels. Additional consideration should be made to starting empiric anticoagulation therapy given the probability of disease in this patient cohort.

In the highest $\mathrm{D}$-dimer category, eliminating patients with suspected DVT who were positive on their Wells' DVT score for recent bedbound/surgery and/or active cancer revealed a prevalence of disease $>50 \%$ in this cohort. Eliminating patients with suspected PE in the highest D-dimer category with a Wells PE score positive for active cancer and/or recent surgery/immobilization also revealed a prevalence of disease $>50 \%$. These data support that in these patients with two common causes of D-dimer elevation to be eliminated, the prevalence of disease remains high. VTE is the most common etiology of D-dimer elevation to that level in this group of patients.

While D-dimer values $<500 \mathrm{ng} / \mathrm{mL}$ have been shown to be highly sensitive for ruling out VTE, the test is not perfect and false-negatives can occur. In the primary study performed, a negative D-dimer had a sensitivity of $98 \%$ for PE and $92 \%$ for DVT. ${ }^{19}$ False-negatives have been associated with increased age of thrombus. One study found subsequent D-dimer values reaching $25 \%$ of initial value of D-dimer VTE by 1 week of symptoms. ${ }^{26}$ Another recommended not utilizing D-dimer testing if clinical symptoms have lasted for greater than 1 week. ${ }^{27} \mathrm{~A}$ prior study suggested that fibroblasts invade old thrombi over time. As a result, fibrin is collagenized and becomes poorly degradable using fibrinolytic enzymes. ${ }^{28}$ Our analysis did not include duration of symptoms as part of data collection. Prior treatment with anticoagulation has also been associated with false-negative D-dimer values. There is also the possibility that normal D-dimer levels may be 
the result of an existing dysfibrinogenemia such as Dusard's syndrome, where the clot formed is poorly degradable, and thus has low D-dimer values. ${ }^{29,30}$

In the 18 patients who had a D-dimer value $<500 \mathrm{ng} / \mathrm{mL}$, but were DVT positive, 9 were found to have an isolated calf DVT. Prior literature has demonstrated that in patients with isolated calf DVTs, the sensitivity and specificity of the Wells DVT score was low at 47 and 74\%, respectively. Compared with DVT-negative patients, however, patients with isolated calf DVTs had higher D-dimer levels. ${ }^{31}$ The diagnosis of isolated calf DVT remains controversial, however.

\section{Limitations}

There were several limitations to this study. First, there were a significant number of patients lost to follow-up after their initial evaluation for VTE. For those found to be PE negative on their index visit, 367 (21.1\%) were lost to follow-up. In those found DVT negative on their index visit, 243 (14.8\%) were lost to follow-up. While every effort was made to limit this number with five phone calls made to attempt followup, nonetheless their ultimate outcomes are not known.

We made the decision to denote isolated subsegmental PE and calf DVT as being VTE positive for the purposes of data analysis. This is an area of controversy as some data support these entities as being false-positive or not needing anticoagulation. $^{32,33}$ Other studies consider these to be VTE events. $^{24,25}$

We used the Innovance D-dimer as our benchmark. While each D-dimer assay has a slightly different performance characteristics, this was likely not a large factor in outcomes, given the high concordance of the Innovance system with the VIDAS D-dimer system. ${ }^{34}$

Finally, due to the small sample size of some of the Ddimer categories, there are wide CIs in some of the groups. We cannot be sure of the specific ORs within each category, but can draw general conclusions given the increasing adjusted ORs.

\section{Future Research and Implications}

This study brings to the forefront the application of the Ddimer in the workup of patients with suspected VTE. Currently used as a primarily dichotomous yes/no test as to whether or not to proceed in VTE evaluation, the association between D-dimer value and likelihood of VTE diagnosis suggests that there is room for subtlety in its application. Our results allow the use of simple bayesian calculators, which allow clinicians to more accurately and specifically estimate a patient's pretest and posttest probabilities of VTE based on D-dimer and imaging results.

Future research can evaluate the patients who are falsenegatives when D-dimer is utilized in suspected VTE. Evaluation of the possible age of thrombus on $\mathrm{D}$-dimer values can be one avenue to explore. It is also possible these patients have abnormal fibrinolytic patterns such as increased levels of $\alpha_{2}$-antiplasmin or have fibrin that is poorly degradable such as in Dusard's syndrome. Additional studies can be aimed toward determining susceptibility of clot formed by clotting plasma in vitro to degradability by fibrinolytic enzymes.

Future research seeking to incorporate D-dimer values or ranges of values into decision instruments may refine the clinician's approach to suspected VTE that increases sensitivity of detecting disease while maintaining appropriate specificity.

Note

Data originally presented at American College of Emergency Physicians (ACEP) Scientific Assembly 2017, Washington DC. Plenary Abstract \#12, October 31, 2017. Abstract title: Highly Elevated Quantitative DDimer Assay Values Increase the Likelihood of Venous Thromboembolism.

Conflict of Interest

None declared.

\section{References}

1 Stein PD, Hull RD, Patel KC, et al. D-dimer for the exclusion of acute venous thrombosis and pulmonary embolism: a systematic review. Ann Intern Med 2004;140(08):589-602

2 Kline JA, Nelson RD, Jackson RE, Courtney DM. Criteria for the safe use of D-dimer testing in emergency department patients with suspected pulmonary embolism: a multicenter US study. Ann Emerg Med 2002;39(02):144-152

3 Oudega R, Moons KG, Hoes AW. Ruling out deep venous thrombosis in primary care. A simple diagnostic algorithm including Ddimer testing. Thromb Haemost 2005;94(01):200-205

4 Ilkhanipour $\mathrm{K}$, Wolfson $\mathrm{AB}$, Walker $\mathrm{H}$, et al. Combining clinical risk with D-dimer testing to rule out deep vein thrombosis. J Emerg Med 2004;27(03):233-239

5 Kristoffersen AH, Ajzner E, Rogic D, et al; joint Working Group on Postanalytical Phase (WG-POST) of the European Federation of Clinical Chemistry and Laboratory Medicine (EFLM) and European Organisation for External Quality Assurance Providers in Laboratory Medicine (EQALM). Is D-dimer used according to clinical algorithms in the diagnostic work-up of patients with suspicion of venous thromboembolism? A study in six European countries. Thromb Res 2016;142:1-7

6 Kabrhel C, Van Hylckama Vlieg A, Muzikanski A, et al. Multicenter evaluation of the YEARS criteria in emergency department patients evaluated for pulmonary embolism. Acad Emerg Med 2018;25(09):987-994

7 McLenachan CJ, Chua O, Chan BS, Vecellio E, Chiew AL. Comparison of Wells and YEARS clinical decision rules with D-dimer for low risk pulmonary embolus patients. Intern Med J 2018; doi: 10.1111/imj.14138

8 Duriseti RS, Brandeau ML. Cost-effectiveness of strategies for diagnosing pulmonary embolism among emergency department patients presenting with undifferentiated symptoms. Ann Emerg Med 2010;56(04):321-332.e10

9 Kabrhel C, Matts C, McNamara M, Katz J, Ptak T. A highly sensitive ELISA D-dimer increases testing but not diagnosis of pulmonary embolism. Acad Emerg Med 2006;13(05):519-524

10 Carrier M, Righini M, Djurabi RK, et al. VIDAS D-dimer in combination with clinical pre-test probability to rule out pulmonary embolism. A systematic review of management outcome studies. Thromb Haemost 2009;101(05):886-892 
11 Murphy N, Broadhurst DI, Khashan AS, Gilligan O, Kenny LC, O'Donoghue K. Gestation-specific D-dimer reference ranges: a cross-sectional study. BJOG 2015;122(03):395-400

12 Righini M, Van Es J, Den Exter PL, et al. Age-adjusted D-dimer cutoff levels to rule out pulmonary embolism: the ADJUST-PE study. JAMA 2014;311(11):1117-1124

13 van der Hulle T, Cheung WY, Kooij S, et al; YEARS study group. Simplified diagnostic management of suspected pulmonary embolism (the YEARS study): a prospective, multicentre, cohort study. Lancet 2017;390(10091):289-297

14 Tick LW, Nijkeuter M, Kramer MH, et al; Christopher Study Investigators. High D-dimer levels increase the likelihood of pulmonary embolism. J Intern Med 2008;264(02):195-200

15 Kohn MA, Klok FA, van Es N. D-dimer interval likelihood ratios for pulmonary embolism. Acad Emerg Med 2017;24(07):832-837

16 Shah K, Quaas J, Rolston D, et al. Magnitude of D-dimer matters for diagnosing pulmonary embolus. Am J Emerg Med 2013;31(06): 942-945

17 Nata S, Hiromatsu S, Shintani Y, Ohno T, Akashi H, Tanaka H. Ddimer value more than $3.6 \mu \mathrm{g} / \mathrm{ml}$ is highly possible existence deep vein thrombosis. Kurume Med J 2013;60(02):47-51

18 Wolf SJ, Hahn SA, Nentwich LM, Raja AS, Silvers SM, Brown MD; American College of Emergency Physicians Clinical Policies Subcommittee (Writing Committee) on Thromboembolic Disease. Clinical Policy: Critical issues in the evaluation and management of adult patients presenting to the emergency department with suspected acute venous thromboembolic disease. Ann Emerg Med 2018;71(05):e59-e109

19 Parry BA, Chang AM, Schellong SM, et al. International, multicenter evaluation of a new D-dimer assay for the exclusion of venous thromboembolism using standard and age-adjusted cutoffs. Thromb Res 2018;166:63-70

20 Yan Z, Ip IK, Raja AS, Gupta A, Kosowsky JM, Khorasani R. Yield of CT pulmonary angiography in the emergency department when providers override evidence-based clinical decision support. Radiology 2017;282(03):717-725

21 Hutchinson BD, Navin P, Marom EM, Truong MT, Bruzzi JF. Overdiagnosis of pulmonary embolism by pulmonary $\mathrm{CT}$ angiography. AJR Am J Roentgenol 2015;205(02):271-277

22 Markel A, Weich Y, Gaitini D. Doppler ultrasound in the diagnosis of venous thrombosis. Angiology 1995;46(01):65-73
23 Hogg KE, Brown MD, Kline JA. Estimating the pretest probability threshold to justify empiric administration of heparin prior to pulmonary vascular imaging for pulmonary embolism. Thromb Res 2006;118(05):547-553

24 Stein PD, Fowler SE, Goodman LR, et al; PIOPED II Investigators. Multidetector computed tomography for acute pulmonary embolism. N Engl J Med 2006;354(22):2317-2327

25 Goodacre S, Sampson F, Thomas S, van Beek E, Sutton A. Systematic review and meta-analysis of the diagnostic accuracy of ultrasonography for deep vein thrombosis. BMC Med Imaging 2005; 5:6

26 D'Angelo A, D'Alessandro G, Tomassini L, Pittet JL, Dupuy G, Crippa L. Evaluation of a new rapid quantitative D-dimer assay in patients with clinically suspected deep vein thrombosis. Thromb Haemost 1996;75(03):412-416

27 Goldin Y, Pasvolsky O, Rogowski O, et al. The diagnostic yield of Ddimer in relation to time from symptom onset in patients evaluated for venous thromboembolism in the emergency medicine department. J Thromb Thrombolysis 2011;31(01):1-5

28 Mirshahi M, Azzarone B, Soria J, Mirshahi F, Soria C. The role of fibroblasts in organization and degradation of a fibrin clot. J Lab Clin Med 1991;117(04):274-281

29 Soria J, Soria C, Caen P. A new type of congenital dysfibrinogenaemia with defective fibrin lysis-Dusard syndrome: possible relation to thrombosis. Br J Haematol 1983;53(04):575-586

30 Lijnen HR, Soria J, Soria C, Collen D, Caen JP. Dysfibrinogenemia (fibrinogen Dusard) associated with impaired fibrin-enhanced plasminogen activation. Thromb Haemost 1984;51(01): 108-109

31 Sartori M, Cosmi B, Legnani C, et al. The Wells rule and D-dimer for the diagnosis of isolated distal deep vein thrombosis. J Thromb Haemost 2012;10(11):2264-2269

32 Kearon C, Akl EA, Ornelas J, et al. Antithrombotic therapy for VTE disease: CHEST Guideline and Expert Panel Report. Chest 2016; 149(02):315-352

33 Palareti G. Do isolated calf deep vein thrombosis need anticoagulant treatment? J Thorac Dis 2016;8(12):E1691-E1693

34 Salvagno GL, Lippi G, Manzato F, et al. Analytical comparison of AxSYM, HemosIL DD HS and Innovance D-dimer immunoassays with the Vidas D-dimer. Int J Lab Hematol 2009;31(04): 475-477 